\title{
Scientists divided over controversial avian flu research
}

In the past ten years, naturally occurring $\mathrm{H} 5 \mathrm{~N} 1$ avian influenza virus has proven lethal in nearly $60 \%$ of the 583 confirmed cases in humans. This highly deadly strain of the flu is currently being investigated in order to determine how flu viruses evolve from infecting only animals to being transmittable to humans.

Recently, researchers in leading influenza laboratories have found that viruses possessing a specific protein from the highly pathogenic $\mathrm{H} 5 \mathrm{~N} 1$ influenza viruses can become transmissible in ferrets, which are considered the best model for studying the flu because they contract the illness much the way humans do. In the course of the work, the scientists altered flu viruses to make them more transmissible, sparking a public debate over the importance and the dangers of such research. To publish the results of this work in a way that would allow them to be replicated by others could be extremely dangerous. Another fear is that the ferret-transmissible viruses may escape from the laboratories and lead to a flu pandemic.

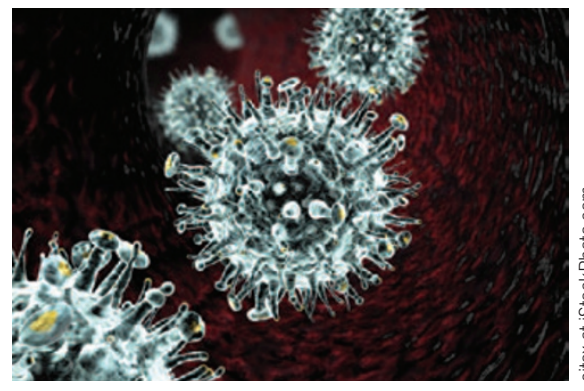

In response to these concerns, Ron A.M. Fouchier of Erasmus MC in Rotterdam (the Netherlands), Adolfo Garcia-Sastre of Mount Sinai School of Medicine (New York, NY) and Yoshihiro Kawaoka of the University of Tokyo (Japan) and the University of Wisconsin (Madison) have agreed to voluntarily halt any research involving the generation of viruses from the avian flu $\mathrm{H} 5 \mathrm{~N} 1$ viruses that are more transmissible in mammals (Nature doi:10.1038.481443A; published online 20 January 2012). The researchers state that they hope the moratorium will provide time for scientists to "clearly explain the benefits of this important research and the measures taken to minimize possible risks... We realize that organizations and governments around the world need time to find the best solutions for opportunities and challenges that stem from the work."

Little is known about what makes an influenza virus transmissible in humans, and this lack of understanding has been a major obstacle in preventing influenza pandemics. Scientists disagree, however, on whether research such as the work with $\mathrm{H} 5 \mathrm{~N} 1$ in ferrets will help prevent a pandemic or potentially help to create one. The research on influenza virus transmission has been conducted in laboratories that use the highest international standards of biosafety and biosecurity practices to prevent the release of transmissible viruses. Despite these precautions, the scientific community remains concerned. Several scientists selected by the World Health Organization will consider what the next steps should be at an international meeting next month.

\section{Kara Rosania}

\section{WHOLELOTTA SOUEAKIN' GOIN' ON}

Like many rodents, male mice (Mus musculus) produce ultrasonic vocalizations (USVs) when they encounter females or the scent of females. The squeaks are attractive to female mice, which can discriminate between calls of siblings and those of unrelated males. These characteristics suggest that the 'songs' play a part in courtship and mating, but their content and function have remained something of a mystery.

To find out what all the squeaking is about, Frauke Hoffmann and colleagues Kerstin Musolf and Dustin J. Penn at the Konrad Lorenz Institute of Ethology, Veterinary University of Vienna (Austria) decided to listen in on the USVs of wild mice. They recorded the vocalizations of 15 adult male offspring of wild-caught mice after exposing them to the scent of female mice. Three of the males did not produce any vocalizations at all, but the recordings from the remaining 12 mice were slowed down and analyzed spectrographically.

The analysis showed that the USVs had several features resembling a song. USVs could be classified by both duration and frequency

(J. Ethol. 30, 173-180; 2012), with frequency being the clearest distinguishing characteristic. The vocalizations included four main types of syllables: high-frequency, low-frequency, one-frequency step and two-frequency step. Males emitted more high-frequency syllables, which were shorter and higher in pitch, than low-frequency syllables when exposed to the scent of females. Furthermore, the wild males seemed to produce more high-frequency syllables than previously reported for lab mice, a difference that may be related to domestication.

Hoffmann's group also found that the USVs included signals of kinship and of individuality (Physiol. Behav. 105, 766-771; 2012). The 12 vocalizing mice belonged to five sibling groups, and the spectrographic analyses showed that calls could be classified according to kinship. The average rate of correct sibling group classification was $98.6 \%$ for low-frequency syllables and $89.0 \%$ for high-frequency syllables. Calls could also be reliably classified according to individual: the average rate of correct individual classification was $64.3 \%$ for low-frequency syllables and $63.9 \%$ for high-frequency syllables.

"It seems as though house mice might provide a new model organism for the study of song in animals," said Penn in a statement from the Veterinary University of Vienna. "Who would have thought that?"

Further studies may investigate the consistency of individual USVs over time and in different contexts, the factors underlying the similarity of USVs among related males and the reasons why some males do not produce USVs at all. 